\title{
Comparative genomic analysis of a new tellurite-resistant Psychrobacter strain isolated from the Antarctic Peninsula
}

\author{
Claudia Melissa Muñoz-Villagrán ${ }^{1,2}$, Katterinne $\mathbf{N}$ Mendez ${ }^{3}$, Fabian Cornejo ${ }^{1}$, Maximiliano Figueroa ${ }^{1}$, \\ Agustina Undabarrena ${ }^{4}$, Eduardo Hugo Morales ${ }^{1}$, Mauricio Arenas-Salinas ${ }^{5}$, Felipe Alejandro Arenas ${ }^{1}$, \\ Eduardo Castro-Nallar Corresp., 3 , Claudio Christian Vásquez ${ }^{\text {Corresp. } 1}$ \\ 1 Laboratorio de Microbiología Molecular. Departamento de Biología, Universidad de Santiago de Chile, Santiago, Chile \\ 2 Departamento de Ciencias Básicas, Facultad de Ciencia, Universidad Santo Tomas Sede Santiago, Santiago, Chile \\ 3 Center for Bioinformatics and Integrative Biology, Facultad de Ciencias Biológicas, Universidad Andrés Bello, Santiago, Chile \\ 4 Laboratorio de Microbiología Molecular y Biotecnología Ambiental, Departamento de Química \& Centro de Biotecnología Daniel Alkalay Lowitt, \\ Universidad Técnica Federico Santa María, Valparaíso, Chile \\ ${ }^{5}$ Centro de Bioinformática y Simulación Molecular, Universidad de Talca, Talca, Chile \\ Corresponding Authors: Eduardo Castro-Nallar, Claudio Christian Vásquez \\ Email address: eduardo.castro@unab.cl, claudio.vasquez@usach.cl
}

The Psychrobacter genus is a cosmopolitan and diverse group of aerobic, cold-adapted, Gram- negative bacteria exhibiting biotechnological potential for low-temperature applications including bioremediation. Here, we present the draft genome sequence of a bacterium from the Psychrobacter genus isolated from a sediment sample from King George Island, Antarctica (3,490,622 bp; 18 scaffolds; $G+C=42.76 \%)$. Using phylogenetic analysis, biochemical properties and scanning electron microscopy the bacterium was identified as Psychrobacter glacincola BNF20, making it the first genome sequence reported for this species. P. glacincola BNF20 showed high tellurite (MIC $2.3 \mathrm{mM}$ ) and chromate (MIC $6.0 \mathrm{mM}$ ) resistance, respectively. Genome-wide nucleotide identity comparisons revealed that P. glacincola BNF20 is highly similar ( $>90 \%$ ) to other uncharacterized Psychrobacter spp. such as JCM18903, JCM18902, and P11F6. Bayesian multi-locus phylogenetic analysis showed that P. glacincola BNF20 belongs to a polyphyletic clade with other bacteria isolated from Polar Regions. A high number of genes related to metal(loid) resistance were found, including tellurite resistance genetic determinants located in two contigs: Contig LIQB01000002.1 exhibited 5 ter genes, each showing putative promoter sequences (terACDEZ), whereas contig LIQB1000003.2 showed a variant of the terZ gene.

Finally, investigating the presence and taxonomic distribution of ter genes in the NCBI's RefSeq bacterial database (5,398 genomes, as January 2017), revealed that 2,623 $(48.59 \%)$ genomes showed at least one ter gene. At the family level, most (68.7\%) genomes harbored one ter gene and $15.6 \%$ exhibited five (including P. glacincola BNF20). Overall, our results highlight the diverse nature (genetic and geographic diversity) of the 
Psychrobacter genus, provide insights into potential mechanisms of metal resistance, and exemplify the benefits of sampling remote locations for prospecting new molecular determinants. 


\section{Comparative genomic analysis of a new tellurite-resistant Psychrobacter strain isolated from the Antarctic Peninsula}

2 Claudia Melissa Muñoz-Villagrán",5\#, Katterinne N. Mendez ${ }^{2, \#, ~ F a b i a ́ n ~ C o r n e j o ~}{ }^{1}$, 3 Maximiliano Figueroa ${ }^{1}$, Agustina Undabarrena ${ }^{3}$, Eduardo Hugo Morales ${ }^{1}$, Mauricio 4 Arenas-Salinas ${ }^{4}$, Felipe Alejandro Arenas ${ }^{1}$, Eduardo Castro-Nallar ${ }^{2, *}$, Claudio Christian 5 Vásquez ${ }^{1, *}$

$7 \quad{ }^{1}$ Laboratorio de Microbiología Molecular, Universidad de Santiago de Chile, Departamento

8 Química y Biología, Santiago, Chile

$9{ }^{2}$ Center for Bioinformatics and Integrative Biology, Facultad de Ciencias Biológicas,

10 Universidad Andrés Bello, Santiago, Chile

11 32Laboratorio de Microbiología Molecular y Biotecnología Ambiental, Departamento de Química

12 y Centro de Biotecnología Daniel Alkaya Lowitt, Universidad Técnica Federico Santa María,

13 Valparaíso, Chile

$14{ }^{4}$ Centro de Bioinformática y Simulación Molecular, Universidad de Talca, Talca, Chile

15 50partamento de Ciencias Básicas, Facultad de Ciencia, Universidad Santo Tomás, Sede

16 Santiago, Chile

\#authors that contributed equally

*Correspondence to:

Claudio C. Vásquez

claudio.vasquez@usach.cl

Eduardo Castro-Nallar

eduardo.castro@unab.cl 


\section{Abstract}

The Psychrobacter genus is a cosmopolitan and diverse group of aerobic, cold-adapted, Gramnegative bacteria exhibiting biotechnological potential for low-temperature applications including bioremediation. Here, we present the draft genome sequence of a bacterium from the Psychrobacter genus isolated from a sediment sample from King George Island, Antarctica $(3,490,622 \mathrm{bp} ; 18$ scaffolds; $\mathrm{G}+\mathrm{C}=42.76 \%)$. Using phylogenetic analysis, biochemical properties and scanning electron microscopy the bacterium was identified as Psychrobacter glacincola BNF20, making it the first genome sequence reported for this species. P. glacincola BNF20 showed high tellurite (MIC $2.3 \mathrm{mM}$ ) and chromate (MIC $6.0 \mathrm{mM}$ ) resistance, respectively. Genome-wide nucleotide identity comparisons revealed that $P$. glacincola BNF20 is highly similar ( $>90 \%$ ) to other uncharacterized Psychrobacter spp. such as JCM18903, JCM18902, and P11F6. Bayesian multi-locus phylogenetic analysis showed that $P$. glacincola BNF20 belongs to a polyphyletic clade with other bacteria isolated from Polar Regions. A high number of genes related to metal(loid) resistance were found, including tellurite resistance genetic determinants located in two contigs: Contig LIQB01000002.1 exhibited 5 ter genes, each showing putative promoter sequences (ter $A C D E Z$ ), whereas contig LIQB1000003.2 showed a variant of the ter $Z$ gene.

Finally, investigating the presence and taxonomic distribution of ter genes in the NCBI's RefSeq bacterial database (5,398 genomes, as January 2017), revealed that 2,623 (48.59\%) genomes showed at least one ter gene. At the family level, most (68.7\%) genomes harbored one ter gene and $15.6 \%$ exhibited five (including $P$. glacincola BNF20). Overall, our results highlight the diverse nature (genetic and geographic diversity) of the Psychrobacter genus, provide insights into potential mechanisms of metal resistance, and exemplify the benefits of sampling remote locations for prospecting new molecular determinants. 
67 The bacterial genus Psychrobacter was first described by Juni and Heym (Juni and Heym, 1986) and includes a group of non-motile, oxidase positive, psychrotolerant, Gram negative rods or coccobacilli isolated from animals and processed foods (Bozal et al., 2003). Bacteria from the Psychrobacter genus have also been isolated from natural environments such as Antarctic ornithogenic soils, sea ice, deep-sea, and sea water from the Pacific Ocean and other locations (Bowman et al., 1997; Romanenko et al., 2002). Antarctic isolates belonging to the genus Psychrobacter have been described and identified as P. inmobilis, P. glacincola, P. luti and $P$. fozzi (Bozal et al., 2003).

The Antarctic territory is the coldest and driest environment on the planet and is exposed to high levels of UV radiation, which favors the production of intracellular Reactive Oxygen Species (ROS) (D'Amico et al., 2006; Potts, 1994). Consequently, microbial communities residing in the Antarctica are unique and some possess diverse strategies to cope with the deleterious effects of ROS and other extreme conditions. In agreement, several microorganisms resistant to antibiotics and other toxicants including tellurite have been isolated from this environment (Arenas et al., 2014; De Souza et al., 2006; Lo Giudice et al., 2013). Many of these organisms can grow at low temperatures and tolerate/resist different compounds, making them ideal candidates for biotechnological applications such as the production of polyunsaturated fatty acids, bioremediation, or as source of industrially useful enzymes (e.g., proteases, lipases) (Brenchley, 1996; Denner et al., 2001).

Tellurite is extremely harmful to most microorganisms, and its toxicity has been associated with the establishment of an oxidative stress status, including ROS generation (Chasteen et al., 2009; Pérez et al., 2007). These ROS are produced as a byproduct of tellurite reduction to its elemental form by either enzymatic or non-enzymatic mechanisms, as visualized by the accumulation of black deposits near the bacterial membrane (Amoozegar et al., 2008; Chasteen and Bentley, 2003; Taylor et al., 1988). Other metal(oid) resistance mechanisms commonly found in bacteria include global cellular responses, cell grouping, uptake control and oxidative stress response, among others (Lemire et al., 2013).

Some of the genes implicated in tellurite resistance include $\operatorname{trg} A B$ from Rhodobacter sphaeroides (unknown function, encoding likely membrane-associated proteins) (O'Gara et al., 1997), tmp from Pseudomonas syringae (encoding a thiopurine methyltransferase, involved in tellurium alkylation) (Prigent-Combaret et al., 2012), lpdA from Aeromonas caviae ST (encoding dihydrolipoamide dehydrogenase, involved in tellurite reduction) (Castro et al., 2008) and gor from Pseudomonas sp. (encoding glutathione reductase, involved in tellurite reduction) (Arenas et al., 2016; Pugin et al., 2014). Tellurite resistance genes were also identified in the ter operon (terZABCDEF) from Escherichia coli and other pathogenic species (Taylor, 1999; Whelan et al., 1997).

Interestingly, the ter operon is not only associated with tellurite resistance but also with resistance to bacteriophage infections and to antimicrobial compounds like colicins (Whelan et al., 1995). The mechanism of action of the proteins encoded by the ter operon remains to be elucidated; however, it is known that they form a multi-subunit complex associated with the inner surface of the bacterial membrane (Anantharaman et al., 2012). Likewise, only terB, terC, 
108 terD and terE have been shown to be directly involved in tellurite resistance (Taylor et al., 109 2002).

110 During the Chilean Antarctic expedition ECA-48 in 2012, a bacterium -later identified as $P$.

111 glacincola BNF20- was isolated and characterized; although P. glacincola BNF20 was highly

112 resistant to tellurite (MIC $2.3 \mathrm{mM}$ ), it did not show increased ROS levels or tellurite reductase

113 activity when exposed to the toxicant (Arenas et al., 2014). In this work, we determined for the

114 first time the genome sequence of a member of the $P$. glacincola species. To gain insight into the

115 potential mechanism(s) of tellurite resistance, we conducted a comparative genomics analysis

116 using available Psychrobacter genome sequences. Specifically, we tested if P. glacincola BNF20

117 was phylogenetically related to other Psychrobacter Antarctic isolates, and if it harbors ter

118 genes. Finally, the ter gene taxonomic distribution was assessed using a reference database

119 containing over 5,000 bacterial genomes.

120

1212 Materials and methods

122

123

124

125

P. glacincola BNF20 was isolated from a sediment sample collected at King George Island,

126

127

128

129

130

131

\subsection{Strain isolation and culture conditions}

Antarctica (S62 $11^{\prime} 37.6^{\prime \prime}$; W58 56 14.9') during the ECA-48 Chilean Antarctic Expedition (January 2012). Bacteria were grown at $25^{\circ} \mathrm{C}$ as described previously (Arenas et al., 2014) in Lysogenic Broth (LB) medium (Sambrook and Russell, 2001) supplemented with tellurite (200 $\mu \mathrm{g} / \mathrm{ml}$ ). Strains were identified by sequencing the 16S rRNA gene (accession MF806171) and determining the fatty acid profile. The 16S rRNA gene was sequenced at Pontificia Universidad Católica de Chile using Sanger sequencing with the primers 8F (5'AGAGTTTGATCCTGGCTCAG-3') (Turner et al., 1999) and 1492R (5'ACGGCTACCTTGTTACGACTT-3') (Lane, 1991). Fatty acid analyses were carried out at DSMZ, Braunschweig, Germany (Kämpfer and Kroppenstedt, 1996). The strain was deposited at DMSZ (Germany), accession \# 102806.

To determine the minimal inhibitory concentration (MIC), bacteria were grown overnight in LB medium with shaking at $25{ }^{\circ} \mathrm{C}$. Subsequently, saturated cultures were diluted 1:100 with fresh medium and grown to $\mathrm{OD}_{600} \sim 0.4-0.5$. Then, $10 \mu \mathrm{l}$ were added to $990 \mu \mathrm{l}$ of LB medium

139 containing serial dilutions of defined toxicants in 48-well culture plates. The plates were

140 incubated with constant shaking for $24 \mathrm{~h}$ at $25{ }^{\circ} \mathrm{C}$. Assayed toxicants included $\mathrm{K}_{2} \mathrm{TeO}_{3}, \mathrm{~K}_{2} \mathrm{CrO}_{4}$, $141 \mathrm{CdCl}_{2}, \mathrm{ZnCl}_{2}, \mathrm{CuSO}_{4}, \mathrm{HAuCl}_{4}, \mathrm{AgNO}_{3}, \mathrm{NiSO}_{4}, \mathrm{NaAsO}_{2}$ and $\mathrm{Na}_{2} \mathrm{HAsO}_{4}$. 
146 A phylogenetic tree of $P$. glacincola BNF20 -based on the partial 16S rRNA gene sequence- was

147 constructed with bootstrap values based on 1,000 replications (Felsenstein, 1985). The nearly

148 complete 16S rRNA gene sequence (1,516 nt) was obtained by merging the PCR sequenced

149 amplicons (accession MF806171) and the sequence obtained by whole genome shotgun

150 sequencing (accession AMK37_RS07000). Sequence alignments, assembly and comparisons,

151 along with best model calculation and construction of the phylogenetic tree were carried out

152 using the MEGA software version 6.0 (Tamura et al., 2013). As outcome, Jukes Cantor model

153 and Pairwise Deletions for gaps treatment was the best fitting model for these sequence data.

154 Nucleotide sequence positions from 16 to 1,535 were considered, according to the E. coli K-12

155 16S rRNA gene sequence numbering (accession AP012306). Scale bar represents 0.01

156 substitutions per-nucleotide positions. Moraxella osioensis DSM 6998 ${ }^{\mathrm{T}}$ (JN175341) was used as

157 outgroup. The following 16S rRNA sequences from Psychrobacter strains were collected from

158 GenBank (accession numbers are given in parentheses): P. glacincola DSM 12194T

159 (AJ312213); P. adeliensis DSM 15333T (AJ539105); P. urotivorans DSM 14009T (AJ609555);

160 P. arcticus DSM 17307T (AY444822); P. cibarius DSM 16327T (AY639871); P. cryohalolentis

161 DSM 17306T (AY660685); P. frigidicola DSM 12411T (AJ609556); P. fozii NF23T

162 (AJ430827); P. inmobilis DSM 7229T (U39399); P. namhaensis DSM 16330T (AY722805); $P$.

163 aquimaris DSM 16329T (AY722804); P. luti NF11T (AJ430828); P. alimentarius DSM 16065T

164 (AY513645); P. fulvigenes JCM 15525 (AB438958); P. piscatorii JCM 15603 (AB453700); $P$.

165 jeotgalli JCM 11463T (AF441201); P. arenosus DSM 15389T (AJ609273) and P. okhotskensis

166 JCM 11840 (AB094794).

167

168

2.3 Preparation of genomic DNA

169

170

171

172

P. glacincola BNF20 was grown in LB medium at $25^{\circ} \mathrm{C}$ for $24 \mathrm{~h}$ with constant shaking. DNA was extracted using the Wizard Genomic ${ }^{\circledR}$ DNA Purification Kit (Promega). The quality and integrity of gDNA was determined by agarose gel $(1 \%)$ electrophoresis and by determining the

173 $260 / 280 \mathrm{~nm}$ absorbance ratio in a microplate multireader equipment (Tecan Infinite ${ }^{\circledR} \mathrm{PRO}$ ).

174

175

\subsection{Genome sequencing and annotation}

176

The draft genome sequence of $P$. glacincola BNF20 was determined by a whole-genome shotgun

178 strategy using the Illumina HiSeq 2000 platform with a mate-pair library of $3 \mathrm{~kb}$ (Macrogen ${ }^{\circledR}$ ).

179 A total of 10.89 million reads were quality filtered and assembled using the A5 pipeline (Tritt et al., 2012). Open reading frame prediction and annotation was carried out using standard operational procedures (Tanenbaum et al., 2010). Gene models were predicted using Glimmer 3.02 (Salzberg et al., 1998) and predicted coding sequences were annotated by comparison with public databases (COG, PFAM, TIGRFAM, UNIPROT, and NR-NCBI). P. glacincola BNF20 predicted proteome completeness was assessed by the presence/absence of bacterial orthologs according to the OrthoDB database using BUSCO (Simão et al., 2015). The circular genome map was assembled from $P$. glacincola BNF20 GenBank formatted file (NZ_LIQB00000000.1) using the plotMyGBK wrapper script (https://github.com/microgenomics/plotMyGBK); 
188 plotMyGBK uses BioPython and the R platform with the packages rSamTools, OmicCircos, and 189 data.table to produce a vector image of a circular map (Cock et al., 2009; R Development Core

190 Team, 2011; Morgan et al, 2016; Hu et al., 2014; https:/github.com/Rdatatable/data.table).

191

\subsection{Nucleotide sequence accession and culture collection number}

Raw sequence data from P. glacincola BNF20 are available online under the BioProject \# PRJNA293364, and Gold ID Gp0145575. The genome project has been deposited at GenBank, accession number NZ_LIQB00000000. The strain was deposited at the DSMZ culture collection, ID number DSM 102806.

\subsection{Psychrobacter genome dataset}

200

201

A total of 35 Psychrobacter genomes including P. glacincola BNF20 were retrieved from

202 NCBI's Genome and JGI GOLD databases (as of February 2017), where 10 and 24 genomes

203 were annotated to the species and genus level, respectively.

204

\subsection{Phylogenetic relationships and whole-genome nucleotide identity}

The average nucleotide identity (ANI) was calculated for the 35-genome dataset using the pyani Python3 module (Pritchard et al., 2016) and the results were visualized using the data.table and pheatmap R packages (https://github.com/Rdatatable/data.table; https://cran.rproject.org/web/packages/pheatmap/index.html). Thirty-one phylogenetic marker genes corresponding to widespread housekeeping genes $d n a G$, nus $A, r p l A, r p l D, r p l K, r p l N, r p l T, r p s B$, $r p s I, r p s M$, tsf, frr, pgk, rplB, rplE, rplL, rplP, rpmA, rpsC, rpsJ, rpsS infC, pyrG, rplC, rplF, $r p l M, r p l S, r p o B, r p s E, r p s K$, and $\operatorname{smp} B$ were identified in each Psychrobacter genome (AMPHORA2; Wu and Eisen, 2008). Each gene was translated under standard genetic code to perform a protein-coding-guided multiple nucleotide sequence alignment, using TranslatorX MUSCLE for the multiple sequence alignment (Abascal et al., 2010; Edgar, 2004). Alignments were concatenated using the alignment editor tool Seqotron (Fourment and Holmes, 2016) and the best partition scheme and substitution model was evaluated by PartitionFinder2 (Lanfear et al., 2016). Finally, the software MrBayes v3.2 was used for phylogenetic reconstruction (Ronquist et al., 2011), and the resulting tree was plotted and annotated using FigTree v1.4.3 (http://tree.bio.ed.ac.uk/software/figtree/). Phylogenetic tree annotation was based on the geographic location, according to BioSample database information for each Psychrobacter genome.

223

\subsection{Search for metal resistance ortholog genes}


226 To identify metal resistance genes, especially ter genes, a bidirectional Blast analysis was performed using the CRB-BLAST method (https://github.com/cboursnell/crb-blast). The BacMet Metal Resistance database (Pal et al., 2014) was used as target and the 35-genome dataset as query. In addition, each genome was re-annotated using the same methodology to identify syntenic genes based on BacMet and Prokka annotation without the bias of different annotation labels as implemented in Prokka v1.12 (Seemann, 2014). Finally, the results were visualized in their genomic context using the in-house script multiGenomicContext

233 (https://github.com/Sanrrone/multiGenomicContext).

\subsection{Promoter search}

236

237

238

239

240

241

242

\section{3}

244

245

246

247

248

249

250

251

252

253

254

255

256

257

258

259

260

To elucidate if ter genes were under the control of one or more promoters, two promoter prediction tools were used on specific contigs where ter genes were found (LIQB01000002.1, position 189803-204267; LIQB01000003.2, position 286133-301767): PromPredict algorithm and the online program BPROM (Rangannan and Bansal, 2010;

http://www.softberry.com/berry.phtml?topic=bprom\&group=programs\&subgroup=gfindb). Both results were jointly analyzed.

\subsection{Taxonomic classification of ter genes}

To investigate if the presence and number of ter genes was restricted to certain taxonomic levels, we downloaded all the bacterial reference genomes from the NCBI's RefSeq database (January 2017; ftp://ftp.ncbi.nlm.nih.gov/refseq/release/bacteria/) and performed a bidirectional Blast searches (crb-blast) against the protein sequences of all ter genes in the BactMet database. Then, the in-house script fetchMyLineage (https://github.com/Sanrrone/fetchMyLineage) was employed to obtain the complete lineage of each bacterial genome with at least one ter gene match. The results were finally visualized using the R packages: ggplot2, RColorBrewer, devtools, ggjoy purr andreshape2 packages (R Development Core Team, 2011; http://ggplot2.org; https://cran.r-project.org/web/packages/RColorBrewer/index.html; https://github.com/hadley/devtools; https://cran.r-project.org/web/packages/ggjoy/index.html; Wickham, 2007).

\section{$3 \quad$ Results}

\subsection{A new Psychrobacter species from the Chilean Antarctic territory}


261

262

263

264

265

266

267

268

269

270

271

272

273

274

275

276

277

278

279

280

281

282

283

284

285

286

287

288

289

290

291

292

293

294

295

296

297

298

P. glacincola BNF20 was isolated from Antarctic sediments and is a Gram-negative, non-motile, aerobic, oxidase positive, rod-shaped bacterium with an average dimension of $1.66 \mu \mathrm{m}$ length and $1.09 \mu \mathrm{m}$ width (Table 1, Figure 1A). The fatty acid composition was determined at the DSMZ Institute (Germany) and showed that the major fatty acid was cis-9 octadecenoic acid $\mathrm{C}_{18: 1} \omega 9 \mathrm{c}(63.78 \%)$. The morphology description and major fatty acid component agrees with previous studies of Antarctic Psychrobacter isolates (Bozal et al., 2013). Initially, BNF20 was erroneously identified as $P$. inmobilis, based on a partial 16S rRNA gene sequence (Arenas et al., 2014). However, re-sequencing and a phylogenetic analysis of the partial 16S rRNA gene revealed that it is related to the $P$. glacincola species, family Pseudomonadaceae from the Gammaproteobacteria class (Figure 1B). Altogether, morphology (electron microscopy), biochemical properties, partial (Sanger) and full length (NGS) $16 \mathrm{~S}$ rRNA gene sequence analysis, and fatty acid composition suggest that isolate BNF20 is member of the $P$. glacincola species.

\subsection{P. glacincola BNF20 tolerates high tellurite and chromate concentrations}

Several tests were carried out to determine if BNF20 was resistant to multiple metals. Besides tellurite (used in the initial selection), P. glacincola BNF20 was 4 times more resistant to chromate than the sensitive strain E. coli BW25113 under optimal growth conditions (Table 2). However, $P$. glacincola BNF20 growth was impaired in the presence of all other metal(loid)s tested, including $\mathrm{Cu}^{2+}, \mathrm{Cd}^{2+}, \mathrm{Hg}^{2+}, \mathrm{Zn}^{2+}, \mathrm{AuCl}_{4}{ }^{1-}, \mathrm{Ni}^{2+}, \mathrm{AsO}_{4}{ }^{2-}, \mathrm{AsO}_{3}{ }^{1-}$, and $\mathrm{Ag}^{1+}$.

\subsection{First draft genome of Psychrobacter glacincola BNF20}

Previous studies showed that $P$. glacincola BNF20 was highly resistant to tellurite (MIC 2.3 $\mathrm{mM}$, Arenas et al., 2014). Although tellurite reduction is often accompanied by the formation of black deposits of elemental tellurium in resistant organisms, this phenotype was not observed in $P$. glacincola BNF20. To further investigate the mechanism(s) of tellurite resistance in $P$. glacincola BNF20, we sequenced the whole genome in search for genetic determinants implicated in metal(loid) resistance. The assembled genome of $P$. glacincola BNF20 consisted of 3,490,622 bp, 18 scaffolds, with an average $\mathrm{G}+\mathrm{C}$ content of $42.76 \%$ (Figure $1 \mathrm{C}$; NCBI Reference Sequence: NZ_LIQB00000000.1). The predicted proteome scored $100 \%$ completeness according to the presence of highly conserved ortholog genes in bacteria (BUSCO analysis). A set of 47 tRNA genes and one copy of the rRNA operon were also identified. From a total of 2,968 predicted CDS, 2,872 (96.7\%) ORFs matched coding sequences available in public databases, of which 2,515 were assigned (84.7\%) or not (352 CDS, $19.31 \%$ ) to COG categories (NZ_LIQB00000000.1). 
299

300

301

302

303

304

305

306

307

308

309

310

311

312

313

314

315

316

317

318

319

320

321

322

323

324

325

326

327

328

329

330

331

332

333

334

335

3.4 P. glacincola BNF20 is evolutionarily divergent from other Antarctic Psychrobacter isolates

The genome sequence of $P$. glacincola BNF20 was compared to other 34 available genomic sequences by estimating ANI values and performing a multi-locus phylogenetic analysis (Figure 2). Besides $P$. glacincola BNF20, the full dataset was composed of 10 named and 24 unnamed Psychrobacter species, respectively. P. glacincola BNF20 exhibited an average nucleotide identity $>95 \%$ and an alignment fraction of over $80 \%$ with 3 isolates designated as Psychrobacter sp. JCM18903 (GCA_000586475.1), Psychrobacter sp. JCM 18902 (GCA_000586455.1) (Kudo et al., 2014) and Psychrobacter sp. P11F6 (GCA_001435295.1) (Moghadam et al., 2016), of which none was isolated from Antarctica. We did not find any genome comparison against BNF20 of $>96.5 \%$ ANI and $>60 \%$ alignment fraction, which has been suggested as a "genomic boundary" for bacterial species (Figure 2A; Varghese et al., 2015). While some of the available genomes come from Antarctic isolates, none of them showed high ANI values (>90\%): P. aquaticus (85\%; GCA_000471625.1); P. alimentarius $(85 \%$; GCA_001606025.1); P. urativorans (85\%; GCA_001298525.1); TB15 (84\%, GCA_000511655.1), G (86\%, GCA_000418305.1); PAMC 21119 (86\%, GCA_000247495.2); TB2 (84\%, GCA_000508345.1); TB47 (86\%, GCA_000511045.1); TB67 (86\%, GCA_000511065.1) and AC24 (86\%, GCA_00051 1635.1$)$.

Supporting our previous results, multi-locus phylogenetic analysis showed that $P$. glacincola BNF20 is more related to P11F6 (isolated from Tunicate ascidians from the Arctic, Moghadam et al., 2016), JCM 18902 and 18903 (isolated from frozen porpoise Neophocaena phocaenoides, Kudo et al., 2014). Antarctic isolates PAMC21119 and G (from King George Island, Moghadam et al., 2016; Che et al., 2013) belong to a polyphyletic group and do not form a monophyletic clade with P. glacincola BNF20, highlighting the heterogeneous nature of the Psychrobacter genus (Figure 2B). All nodes of the phylogeny were well supported (posterior probability $>0.99$ ).

\subsection{P. glacincola BNF20 encodes multiple metal resistance determinants}

As $P$. glacincola BNF20 was isolated from King George Island sediments, a place where heavy metal contamination has not been previously reported, we searched for genes known to be involved in metal resistance that could explain the observed tellurite and chromate resistance of strain BNF20 (BacMet database; Pal et al., 2014). Type and gene copy number distribution was not uniform in the 35-genome Psychrobacter dataset (Supplementary Table 3). 
336 Specifically, $\sim 100$ genes possibly conferring metal resistance were identified in the genome of

337 P. glacincola BNF20, of which some are related to chromate resistance, including chrL

338 (BAC0361; regulatory protein, involved in chromate resistance), chrR (BAC0538; chromate

339 reductase), $m d r L / y f m O$ (BAC0209; multidrug efflux protein $y f m O$ ) and $r u v B$ (BAC0355; ATP-

340 dependent DNA helicase), and some to tellurite resistance -the so-called ter genes (Whelan and

341 Colleran, 1992), including terA (BAC0386), terC (BAC0388), terD (BAC0389), terE

342 (BAC0390) and terZ (BAC0392) (Figure 3, Supplementary Table 3). Two other genes

343 apparently involved in tellurite resistance, $r u v B$ (BAC0355; ATP-dependent DNA helicase) and

344 pitA (BAC0312; low-affinity inorganic phosphate transporter 1), were also identified.

\subsection{Organization of ter genes in P. glacincola BNF20}

Given that i) tellurite is by far more toxic for bacteria than other metals (Taylor, 1999) and ii) it is scarce in the Earth's crust (Turner et al., 2012), finding tellurite resistance determinants in $P$. glacincola BNF20 was somewhat unexpected. Since to date the presence of ter genes in Antarctic microorganisms has not been reported, we focused the following analyses our study on them.

The ter genes were originally described as part of an E. coli operon exhibiting the terZABCDE structure (Taylor et al., 2002). P. glacincola BNF20 harbors terA, terZ, terE, terC and terD orthologs, but not ter $B$ (Figure 3A); terA shows the opposite transcriptional orientation than the rest of the ter genes, while ter $Z$ is duplicated and is contained in different contigs (Figure 3B). In addition, the expression of all ter genes in $P$. glacincola BNF20 seems to be regulated by individual promoters (PromPredict and BPROM analyses), suggesting that they are organized as a gene cluster rather than as an operon.

Three members of the Psychrobacter genus contained one ter gene [P. phenylpyruvicus (terZ,

361 GCA_000685805.1), P. lutiphocae (terZ, GCA_000382145.1) and P. sp. ENNN9 III (terD,

362 GCA001462175.1)], while the rest had different combinations of them (Supplementary Figure

$3631)$.

364 In P. glacincola BNF20, the context of the ter gene cluster is similar to other isolates like

365 Psychrobacter sp. JC18902 (GCA_00058655.1), Psychrobacter sp. G (GCA_000418305.1),

366 Psychrobacter sp. TB67 (GCA_000511065.1), Psychrobacter sp. AC24 (GCA_000511635.1),

367 Psychrobacter sp. TB47 (GCA_00051045.1) and P. arcticus 273-4 (GCA_000012305.1).

368 Interestingly, in all analyzed Psychrobacter genomes the ter gene cluster also contains a gene

369 encoding a protein of the TIGR00266 family (unknown function, Supplementary Figure 1).

370

\section{7 ter genes are distributed over several bacterial Phyla}


373 To determine the frequency of ter genes in known bacterial genomes, their taxonomic

374 distribution was evaluated. In general, ter genes are more commonly found in Gram positive than

375 in Gram negative bacteria. Using NCBI's RefSeq database (> 5,000 genomes; accessed January

376 2017), we found that $48.59 \%$ of them contained ter genes (26 out of 30 bacterial Phyla). While,

377 at the genus level, most genomes had one ter gene (67.95\%) (Supplementary Table 4, Figure 4),

378 others harbor $2(2.31 \%), 3(0.69 \%), 4(5.24 \%), 6(4.61 \%)$ or $7(1.15 \%)$ ter genes. Interestingly,

379 the second most abundant combination of ter genes in genomes was $5(18.04 \%)$, which could

380 suggest evolutionary constrains.

381

382

383

384

385

386

387

388

389

390

391

392

393

394

395

396

397

398

399

400

401

402

403

404

405

406

407

408

409

410

411

412

At the phylum level most Proteobacteria contain one ter gene, with a few exceptions showing up to 7, including Yersiniacee, Morganellaceae, Enterobacteriaceae and Erwiniaceae. A similar pattern is observed in other Phyla, except for Firmicutes where genomes exhibit a defined array of ter genes (Figure 4). Interestingly, while members belonging to the best represented family in RefSeq, i.e., Streptomycetaceae (149 genomes) exhibit 5 or 6 ter genes, in other well-represented families such as Flavobactericidae only 26 out of 114 genomes exhibit 5 ter genes (23\%). Within the Moraxelaceae family, 9 out of 45 genomes show 5 ter genes $(20 \%$, including BNF 20), which agrees with the complete family database distribution ( $18 \%$ with 5 ter genes).

\section{Discussion}

Here we show for the first time the genome sequence of a $P$. glacincola species isolated from Antarctica, which can tolerate high concentrations of tellurite and chromate. P. glacincola BNF20 showed to be 4- and 500-fold more resistance to chromate and the tellurium oxyanion tellurite than E. coli BW25113 (Table 2).

Previous studies showed that defined toxicants can trigger common responses or repair mechanisms (Miranda et al., 2005), suggesting that tellurite and chromate resistance could be related. Besides tellurite and chromate, P. glacincola BNF20 genome encodes resistance determinants associated to a number of other heavy metal(loid)s such as arsenic, cadmium, copper and mercury (Supplementary Table 3). Interestingly, tellurite resistance in P. glacincola BNF20 did not correlate with a strong tellurite reduction, as previously reported (Arenas et al., 2014), which prompted us to search for genes associated with tellurite resistance in its genome. Identifying these genetic resistance determinants could be useful as the Psychrobacter genus has been proposed as good candidate for biotechnological applications including bioremediation (Lasa and Romalde, 2017).

Members of the Psychrobacter genus are versatile and have been isolated from different places with low temperatures -including Antarctica- as well as from some animal hosts including skin, fish gills and guts and human blood, among others (Bowman et al., 1997; Bozal et al., 2003; Romanenko et al., 2002). However, isolates from similar environments show high genomic variability, as evidenced by ANI analysis (Supplementary Table 2). A multi-locus phylogenetic analysis revealed that Antarctic Psychrobacter isolates do not form a monophyletic group (Figure 2). In this context, the presence of ter genes is correlated to some extent with their 
413 genomic structure. In fact, higher ANI values reflected a more similar ter gene organization. 414 Thus, $P$. glacincola BNF20 exhibited a very close ter gene organization with the three closest 415 members Psychrobacter sp. P11F6, JCM18902 and JCM18903 (Figure 2, Supplementary Figure 416 1).

417 Psychrophilic and psychrotolerant microorganisms require several genes to increase their 418 phenotypic flexibility to survive in extreme environments such as cold habitats. Thus, in addition 419 to genes associated with cold shock proteins, membrane fluidity, among others, the presence of 420 metal(loid) resistance genes seems to favor their adaptation (Dziewit and Bartosik, 2014; Rodríguez-Rojas et al., 2016). This is also the case of P. glacincola BNF20, which harbors over 100 putative metal resistance genes (Supplementary Table 2). In principle and even though this high number of genes predicted bacterial resistance to a number of metal(loid)s, MIC determinations showed that $P$. glacincola BNF20 was only resistant to chromate and tellurite (MIC 6 and $2.3 \mathrm{mM}$, respectively). Chromate resistance genes included chrI (regulatory protein of Ralstonia metallidurans CH34; Juhnke et al., 2002), chrR (encoding a chromate reductase; Park et al., 2000), $m d r L / y f m O$ (multidrug efflux transporter in Listeria monocytogenes; Mata et al., 2000) and $r u v B$, encoding a DNA helicase involved in both chromate and tellurite resistance in P. aeruginosa PAO1 (Miranda et al., 2005). Genes related to tellurite resistance identified in P. glacincola BNF20 included the phosphate transporter pitA (Elías et al., 2012) and a cluster of ter genes (Whelan et al., 1995), composed of terA, terZ, terC, terD and terE, which exhibit a different organization as compared to other ter gene clusters previously described (Figure 3). Although ter refers to tellurite resistance, the same genes participate in resistance to phages, colicins (Whelan et al., 1997) and to other oxidative stress-generating antimicrobials (Taylor, 1999), which could be the result of transcriptional control by a common regulator, OxyR (Ni et al., 2014).

A number of reasons may explain the observed discordances among MIC values (i.e., $\mathrm{Hg}, \mathrm{Cu}$, As, etc.) and the respective resistance genes identified in this bacterium. For instance, $P$. glacincola BNF20 sensitivity to mercury could be a result of the absence of some genes (i.e. $m e r \mathrm{~T}$ ) belonging to the mer operon, which could render it non-functional (Boyd and Barkay, 2012). Similarly, the absence of the cus $\mathrm{S}$ gene (Cu sensor) in the P. glacincola BNF20 genome could be responsible for its copper sensitivity, in spite the presence of other genes that participate in $\mathrm{Cu}$ homeostasis (Rensing and Grass, 2003).

444 Tellurite resistance-associated ter genes are grouped in three different families: i) TerC, encompassing transmembrane proteins, ii) TerD, which includes the cytoplasmatic paralogs TerD, TerA, TerE, TerF and TerZ (Anantharaman y cols., 2012), and iii) TerB, representing proteins that are directly associated with the inner surface of the cell membrane, although they also have a cytoplasmatic localization (Alekhina et al., 2011). As mentioned, TerC interacts with TerD, TerB and other proteins showing different cell functions (Turkovicova et al., 2016).

Most bacteria carrying ter genes display a similar transcriptional organization. Thus terZABCDEF, terZABCDE and TerABD present in E. coli $\mathrm{O} 157: \mathrm{H} 7$, Proteus sp. and D. radiodurans, respectively, are operons (Makarova et al., 2001; Ponnusamy and Clinkenbeard, 2015; Taylor et al., 2002). The Psychrobacter genus represents an exception to this rule, with terA lying in the opposite transcriptional orientation (Supplementary Figure 1). 
455 Transcriptomic and proteomic assays have shown that $\operatorname{ter} B$ is expressed when $E$. coli or $D$.

456 radiodurans are exposed to tellurite (Ananganti et al., 2015; Taylor et al., 2002). TerB seems to 457 be essential for tellurite resistance and interacts with some cytoplasmatic proteins such as the 458 alpha subunit of ATP synthase, G subunit of the NADH-dependent quinone oxidoreductase and 459 DnaK chaperone, among others (Alekhina et al., 2011). Given that P. glacincola BNF20 lacks $460 \operatorname{ter} B$, we hypothesize that there must be another gene product that mediates tellurite resistance.

461 Based on their genetic background, ter genes have also been classified into different groups (I to 462 IV) (Anantharaman et al., 2012). In this context and given its similitude with the ter genes found

463

464

465

466

467

468

469

470

471

472

473

474

475

476

477

478

479

480

481

482

483

484

485

486

487

488

489

490

491

492

493

494

495

in Psychrobacter sp. PRwf-1, P. glacincola BNF20 would belong to group I, which contains a gene encoding a protein exhibiting the AIM24 domain, also found in the P. glacincola BNF20 TIGR00266 protein. Although no role has been ascribed to it in prokaryotes, in higher organisms it is an internal membrane protein related to mitochondrial biogenesis which is required for yeast respiration (Deckers et al., 2014). The AIM24 domain exhibits a double beta-helix folding, which is frequently found in genes neighboring TerD, suggesting that both proteins could interact (Anantharaman et al., 2012).

Deciphering the origins of bacterial operons is not straightforward, and there are some hypotheses that try to explain their formation. An interesting example is the piecewise model, which states that the his operon (hisGDCBHAFIE) was gradually formed. Phylogenetic analyses of the Proteobacterial phylum his genes showed their progressive grouping, which suggests that they were located in nearby zones of the chromosome in closely related microorganisms. Following, new events ended with the formation of the hisBHAF central core and the whole operon (Fani et al., 2015).A future hypothesis to test is whether the ter operon has a similar evolutionary origin.

To evaluate the taxonomical distribution of ter genes in the Bacterial kingdom, the 5,398 genomes retrieved from the NCBI's RefSeq bacterial database were screened. About $48.6 \%$ of them $(2,623$ genomes) were found to contain ter genes. While at the family level most (68.7\%) harbored one ter gene (chiefly terC) and $15.6 \%$ exhibited five (including $P$. glacincola BNF20), at the class level the number of genomes exhibiting at least one ter gene was Gammaproteobacteria (379), Alphaproteobacteria (253) and Bacilli (247). Finally and regarding phyla, Proteobacteria, Actinobacteria and Firmicutes had 867, 854 and 361 genomes containing at least one ter gene, respectively (Figure 4, Supplementary Table 4).

Within the Proteobacteria phylum, most families had only one ter gene, while others up to 7 (Morganellaceae, Yersiniaceae), 6 (Chromatiacceae, Budviciaceae), 5 (Moraxellceae, Burkholderiaceae), 4 (Erythrobacteraceae), etc. (Figure 4). In this context, it would be interesting to carry out phylogenetic analyses to understand the evolution of these ter genes and how the currently known terZABCDEF operon was formed (Taylor, 1999; Whelan et al., 1997).

Finally, it was found that -in general- Gram positive microorganisms contain more ter genes than Gram negative bacteria (Supplementary Table 4). This is interesting because it is generally accepted that they also show higher tellurite resistance (Taylor, 1999). For instance, Streptomyces and Bacillus genera comprise 137 and 65 genomes carrying up to 5-6 ter genes, respectively, suggesting that ter gene copy number could be related to the high resistance to 
496 tellurite observed in S. coelicolor and Geobacillus stearothermophilus (Moscoso et al., 1998;

497 Sanssouci et al., 2011).

498

499

\section{Conclusions}

500

501 A new species of Antarctic bacteria exhibiting high tellurite resistance was isolated and 502 identified as $P$. glacincola BNF20. Although within the genus the percent of sequence coverage 503 is low, its genomic sequence is similar to other uncharacterized genomes and contains a large 504 number of genes implicated in metal(loid) resistance, especially chromate and tellurite. The 505 transcriptional orientation of tellurite resistance (ter) genes in P. glacincola BNF20 is different to 506 that described in other microorganisms and most likely do not function as an operon. The wide 507 distribution of ter genes in the bacterial world suggests that they play an important physiological 508 role.

509

510

\section{Acknowledgments}

511 We would like to thank Fraunhöfer Foundation for carrying out the novo assembly and gene 512 annotation, especially to Paz Tapia and Jorge Valdés. We also thank Natalia Valdés from 513 Universidad de Santiago de Chile for helping with the bioinformatic analysis. ECN thanks the 514 high- performance computing facility from The George Washington University, Colonialone, for 515 providing data storage, support, and computing power for genomic analyses

516 (colonialone.gwu.edu).

\section{References}

Abascal F, Zardoya R, Telford MJ. 2010. TranslatorX: multiple alignments of nucleotide sequences guided by amino acid translations. Nucleic Acids Research 38:W7-W13. doi:

521 10.1093/nar/gkq291.

522 Alekhina O, Valkovicova L, Turna J. 2011. Study of membrane attachement and in vivo co523 localization of TerB protein from uropathogenic Escherichia coli KL53. General Physiology and 524 Biophysics 30(3):286-292. doi: 10.4149/gpb_2011_03_286.

525 Amoozegar MA, Ashengroph M, Malekzadeh F, Razavi MR, Naddaf S, Kabiri M. 2008.

526 Isolation and initial characterization of the tellurite reducing moderately halophilic bacterium,

527 Salinicoccus sp. strain QW6. Microbiological Research. 163(4):456-465. doi:

528 10.1016/j.micres.2006.07.010. 
529 Anaganti N, Basu B, Gupta A, Joseph D, Apte SK. 2015. Depletion of reduction potential and

530 key energy generation metabolic enzymes underlies tellurite toxicity in Deinococcus

531 radiodurans. Proteomics 15(1):88-97. doi: 10.1002/pmic.201400113.

532 Anantharaman V, Iyer LM, Aravind L. 2012. Ter-dependent stress response systems: novel 533 pathways related to metal sensing, production of a nucleoside-like metabolite, and DNA534 processing. Molecular BioSystems 8(12):3142-3165. doi: 10.1039/c2mb25239b.

535 Arenas F, Pugin B, Henríquez N, Arenas M, Díaz W, Pozo F, Muñoz CM, Chasteen TG, Pérez536 Donoso JM, Vásquez CC. 2014. Isolation, identification and characterization of highly tellurite537 resistant, tellurite-reducing bacteria from Antarctica. Polar Science 8(1):40-52. doi:

538 10.1016/j.polar.2014.01.001.

539 Arenas M, Vargas-Pérez J, Morales W, Pinto C, Muñoz-Díaz P, Cornejo F, Pugin B, Sandoval 540 JM, Díaz-Vásquez WA, Muñoz-Villagrán C, Rodríguez-Rojas F, Morales EH, Vásquez CC,

541 Arenas FA. 2016. Flavoprotein-mediated tellurite reduction: structural basis and applications to 542 the synthesis of tellurium-containing nanostructures. Frontiers in Microbiology 7:1160.

543 doi:10.3389/fmicb.2016.01160.

544 Bowman JP, Nichols DS, McMeekin TA. 1997. Psychrobacter glacincola sp. nov., a 545 halotolerant, psychrophilic bacterium isolated from Antarctic sea. Systematic and Applied 546 Microbiology 20(2):209-215. doi.org/10.1016/S0723-2020(97)80067-7.

547 Boyd ES, Barkay T. 2012. The mercury resistance operon: from an origin in a geothermal 548 environment to an efficient detoxification machine. Frontiers in Microbiology 3: 349. doi: 549 10.3389/fmicb.2012.00349

550 Bozal N, Montes MJ, Tudela E, Guinea J. 2003. Characterization of several Psychrobacter 551 strains isolated from Antarctic environments and description of Psychrobacter luti sp. nov. and 552 Psychrobacter fozii sp. nov. International Journal of Systemaic and Evolutionary Microbiology 553 53(Pt 4):1093-1100. doi: 10.1099/ijs.0.02457-0.

554 Brenchley JE. 1996. Psychrophilic microorganisms and their cold-active enzymes. Journal of 555 Industrial Microbiology. 17(5-6):432-437. doi: https://doi.org/10.1007/BF01574774.

556 Castro ME, Molina R, Díaz W, Pichuantes SE, Vásquez CC. 2008. The dihydrolipoamide 557 dehydrogenase of Aeromonas caviae ST exhibits NADH dependent tellurite reductase activity. 558 Biochemical and Biophysical Research Communications 375(1):91-94. doi:

559 10.1016/j.bbrc.2008.07.119.

560 Chasteen TG, Bentley R. 2003. Biomethylation of selenium and tellurium: microorganisms and 561 plants. Chemical Reviews 103(1):1-26. doi: 10.1021/cr010210+.

562 Chasteen TG, Fuentes DE, Tantaleán JC, Vásquez CC. 2009. Tellurite: history, oxidative stress, 563 and molecular mechanisms of resistance. FEMS Microbiology Reviews 33(4):820-832. doi:

564 10.1111/j.1574-6976.2009.00177.x. doi: 10.1021/cr010210+. 
565 Che S, Song L, Song W, Yang M, Liu G, Lin X. 2013. Complete genome sequence of antarctic

566 bacterium Psychrobacter sp. strain G. Genome Announcements 1(5):pii:e00725-13. doi:

567 10.1128/genomeA.00725-13.

568 Cock P, Antao T, Chang J, Chapman B, Cox C, Dalke A, Friedberg I, Hamelryck T, Kauff F,

569 Wilczynski B, de Hoon MJ. 2009. Biopython: freely available Python tools for computational

570 molecular biology and bioinformatics. Bioinformatics 25(11):1422-1423. doi:

571 10.1093/bioinformatics/btp163.

572 D'Amico S, Colling T, Marx JC, Felle G, Gerdar C. 2006. Psychrophilic microorganisms:

573 challenges for life. EMBO Reports 7(4):385-389. doi: 10.1038/sj.embor.7400662.

574 De Souza MJ, Nair S, Loka Bharathi PA, Chandramohan D. 2006. Metal and antibiotic-

575 resistance in psychrotrophic bacteria from Antarctic marine waters. Ecotoxicology

576 15(4):379-384. doi: 10.1007/s10646-006-0068-2.

577 Deckers M, Balleininger M, Vukotic M, Römpler K, Bareth B, Juris L, Dudek J. 2014. Aim24

578 stabilizes respiratory chain supercomplexes and is required for efficient respiration. FEBS Letters

579 588(17):2985-2992. doi: 10.1016/j.febslet.2014.06.006.

580 Denner EBM, Mark B, Busse HJ, Turkiewicz M, Lubitz W. 2001. Psychrobacter proteolyticus

581 sp. nov., a psychrotrophic, halotolerant bacterium isolated from the Antarctic krill Euphausia

582 superba Dana, excreting a cold-adapted metalloprotease. Systematic and Applied Microbiology

583 24(1):44-53. doi: 10.1078/0723-2020-00006.

584 Dziewit L, Bartosik D. 2014. Plamids of psychrophilic and psychrotolerant bacteria and their

585 role in adaptation to cold environments. Frontiers in Microbiology 5:596. doi:

$58610.3389 /$ fmicb.2014.00596.

587 Edgar RC. 2004. MUSCLE: multiple sequence alignment with high accuracy and high

588 throughput. Nucleic Acids Research 32(5):1792-1797. doi: 10.1093/nar/gkh340.

589 Elías AO, Abarca MJ, Montes RA, Chasteen TG, Pérez-Donoso JM, Vásquez CC. 2012.

590 Tellurite enters Escherichia coli mainly through the PitA phosphate transporter.

591 MicrobiologyOpen 1(3):259-267. doi: 10.1002/mbo3.26.

592 Fani R, Brilli M, Liò P. 2005. The origin and evolution of operons: the piecewise building of the 593 proteobacterial histidine operon. Journal of Molecular Evolution 60(3):378-390. doi:

594 10.1007/s00239-004-0198-1.

595 Felsenstein J. 1985. Confidence limits on phylogenies: an approach using the bootstrap.

596 Evolution 39(4):783-791. doi:10.2307/2408778.

597 Fourment M, Holmes EC. 2016. Seqotron: a user-friendly sequence editor for Mac OS X. BMC

598 Research Notes 9:106. doi: 10.1186/s13104-016-1927-4. 
599 Hu Y, Yan C, Hsu C, Chen Q, Niu K, Komatsoulis G, Meerzaman D. 2014. OmicCircos: a 600 simple-to-use R package for the circular visualization of multidimensional omics data. Cancer 601 Informatics 13:13-20. doi: 10.4137/CIN.S13495.

602 Juhnke S, Peitzsch N, Hübener N, Große C, Nies DH. 2002. New genes involved in chromate 603 resistance in Ralstonia metallidurans strain CH34. Archives of Microbiology 179(1):15-25. doi: 604 10.1007/s00203-002-0492-5.

605 Juni E, Heym GA. 1986. Psychrobacter immobilis gen. nov., sp. nov.: genospecies composed of 606 Gram-negative, aerobic, oxidase-positive coccobacilli. International Journal of Systematic 607 Bacteriology 36:388-391. doi: 10.1099/00207713-36-3-388.

608 Kämpfer P, Kroppenstedt RM. 1996. Numerical analysis of fatty acid patterns of coryneform 609 bacteria and related taxa. Canadian Journal of Microbiology 42(10):989-1005. doi:

610 https://doi.org/10.1139/m96-128.

611 Kudo T, Kidera A, Kida M, Kawauchi A, Shimizu R, Nakahara T, Zhang X, Yamada A, Amano 612 M, Hamada Y, Taniyama S, Arakawa O, Yoshida A, Oshima K, Suda W, Kuwahara H, Nogi Y, 613 Kitamura K, Yuki M, Iida T, Moriya S, Inoue T, Hongoh Y, Hattori M, Ohkuma M. 2014. Draft 614 genome sequences of Psychrobacter strains JCM 18900, JCM 18901, JCM 18902, and JCM 615 18903, isolated preferentially from frozen aquatic organisms. Genome Announcements 2:e0280616 14. doi: 10.1128/genomeA.00280-14.

617 Lane DJ. 1991. 16S/23S rRNA sequencing. In Nucleic Acid Techniques in Bacterial Systematics. 618 Stackebrandt E, Gooffellow M., eds., pp. 115-175.

619 Lanfear R, Frandsen PB, Wright AM, Senfeld T, Calcott B. 2016. PartitionFinder 2: new 620 methods for selecting partitioned models of evolution for molecular and morphological 621 phylogenetic analyses. Molecular Biology and Evolution 34(3):772-773. doi:

$62210.1093 / \mathrm{molbev} / \mathrm{msw} 260$.

623 Lasa A, Romalde JL. 2017. Genome sequence of three Psychrobacter sp. strains with potential 624 applications in bioremediation. Genomics Data 12:7-10. doi: 10.1016/j.gdata.2017.01.005.

625 Lemire JA, Harrison JJ, Turner RJ. 2013. Antimicrobial activity of metals: mechanisms, 626 molecular targets and applications. Nature Reviews Microbiology 11(6):371-384. doi:

627 10.1038/nrmicro3028.

628 Lo Giudice A, Casella P, Bruni V, Michaud L. 2013. Response of bacterial isolates from 629 Antarctic shallow sediments towards heavy metals, antibiotics and polychlorinates biphenyls.

630 Ecotoxicology 22(2):240-250. doi: 10.1007/s10646-012-1020-2.

631 Makarova KS, Aravind L, Wolf YI, Tatusov RL, Minton KW, Koonin EV, Daly MJ. 2001.

632 Genome of the extremely radiation-resistant bacterium Deinococcus radiodurans viewed from 633 the perspective of comparative genomics. Microbiology and Molecular Biology

634 Reviews 65(1):44-79. doi: 10.1128/MMBR.65.1.44-79.2001. 
635 Mata MT, Baquero F, Pérez-Díaz JC. 2000. A multidrug efflux transporter in Listeria

636 monocytogenes. FEMS Microbiology Letters 187(2):185-188. doi: 10.1111/j.1574-

637 6968.2000.tb09158.x.

638 Miranda AT, González MV, González G, Vargas E, Campos-García J, Cervantes C. 2005.

639 Involvement of DNA helicases in chromate resistance by Pseudomonas aeruginosa PAO1.

640 Mutation Research 578(1-2):202-209. doi: 10.1016/j.mrfmmm.2005.05.018.

641 Moghadam MS, Albersmeier A, Winkler A, Cimmino L, Rise K, Hohmann-Marriot MF, 642 Kalinowski J, Rückert C, Wentzel A, Lale R. 2016. Isolation and genome sequencing of four 643 Arctic marine Psychrobacter strains exhibiting multicopper oxidase activity. BMC Genomics 644 17:117.doi: 10.1186/s12864-016-2445-4.

645 Morgan M, Pagès H, Obenchain V, Hayden N. 2016. Rsamtools: Binary alignment (BAM), 646 FASTA, variant call (BCF), and tabix file import. R package version 1.24.0. Available at 647 http://bioconductor.org/packages/release/bioc/html/Rsamtools.html. doi:

648 10.18129/B9.bioc.Rsamtools.

649 Moscoso H, Saavedra C, Loyola C, Pichuantes S, Vásquez CC. 1998. Biochemical 650 characterization of tellurite-reducing activities of Bacillus stearothermophilus $\mathrm{V}$. Research in 651 Microbiology 149(6):389-397. doi: https://doi.org/10.1016/S0923-2508(98)80321-5.

652 Ni B, Zhang Y, Huang X, Yang R, ZhouD. 2014. Transcriptional regulation mechanism of ter 653 operon by OxyR in Yersinia pestis. Current Microbiology 69(1):42-46. doi:

654 https://doi.org/10.1007/s00284-014-0550-7.

655 O'Gara JP, Gomelsky M, Kaplan S. 1997. Identification and molecular genetic analysis of 656 multiple loci contributing to high-level tellurite resistance in Rhodobacter sphaeroides 2.4.1.

657 Applied and Environmental Microbiology 63(12):4713-4720.

658 Pal C, Bengtsson-Palme J, Rensing C, Kristiansson E, Joakim Larsson DG. 2014. BacMet:

659

660

661 Park CH, Keyhan M, Wielinga B, Fendorf S, Matin A. 2000. Purification to homogeneity and 662 characterization of a novel Pseudomonas putida chromate reductase. Applied and Environmental 663 Microbiology 66(5):1788-1795. doi: 10.1128/AEM.66.5.1788-1795.2000.

664 Pérez JM, Calderón IL, Arenas FA, Fuentes DE, Pradenas GA, Fuentes EL, Sandoval JM, Castro 665 ME, Elías AO, Vásquez CC. 2007. Bacterial toxicity of potassium tellurite: unveiling an ancient 666 enigma. PLoS ONE 2(2):e211. doi: 10.1371/journal.pone.0000211.

667 Ponnusamy D, Clinkenbeard KD. 2015. Role of tellurite resistance operon in filamentous growth 668 of Yersinia pestis in macrophages. PLoS ONE 10(11):e0141984. doi:

669 10.1371/journal.pone.0141984.

670 Potts M. 1994. Dessication tolerance of prokaryotes. Microbiological Reviews 58(4):755-805. 
671 Prigent-Combaret C, Sanguin H, Champier L, Bertrand C, Monnez C, Colinon C, Blaha D, 672 Ghigo JM, Cournoyer B. 2012. The bacterial thiopurine methyltransferase tellurite resistance 673 process is highly dependent upon aggregation properties and oxidative stress response.

674 Environmental Microbiology 14(10):2645-2660.

675 Pritchard L, Glover RH, Humphris S, Elphinstone JG, Toth IK. 2016. Genomics and taxonomy 676 in diagnostics for food security: soft-rotting enterobacterial plant pathogens. Analytical Methods 677 8:12-24. doi: 10.1039/C5AY02550H.

678 Pugin B, Cornejo F, Muñoz-Díaz P, Muñoz-Villagrán C, Vargas-Pérez J, Arenas FA, Vásquez 679 CC. 2014. Glutathione reductase-mediated synthesis of tellurium-containing nanostructures 680 exhibiting antibacterial properties. Applied and Environmental Microbiology 80(22):7061-7070. 681 doi: 10.1128/AEM.02207-14.

682 R Development Core Team. 2011. R: A language and environment for statistical computing. 683 Vienna, Austria: the R Foundation for Statistical Computing. ISBN: 3-900051-07-0.

684 Rangannan V, Bansal M. 2010. High-quality annotation of promoter regions for 913 bacterial 685 genomes. Bioinformatics 26(24):3043-50. doi: 10.1093/bioinformatics/btq577.

686 Rensing C, Grass G. 2003. Escherichia coli mechanism of copper homeostasis in a changing 687 environment. FEMS Microbiology Reviews 27(2-3): 197-213. doi: 10.1016/S0168688 6445(03)00049-4

689 Rodríguez-Rojas F, Díaz-Vásquez W, Undabarrena A, Muñoz-Díaz P, Arenas F, Vásquez, C. 690 2016. Mercury-mediated cross-resistant to tellurite in Pseudomonas spp. isolated from the 691 Chilean Antarctic territory. Metallomics 8(1):108-117. doi: 10.1039/c5mt00256g.

692 Romanenko LA, Schumann P, Rohde M, Lysenko AM, Mikhailov V, Stackebrandt E. 2002.

693 Psychrobacter submarinus sp. nov. and Psychrobacter marincola sp. nov., psychrophilic 694 halophiles from marine environments. International Journal of Systematic and Evolutionary 695 Microbiology 52(Pt 4):1291-1297.

696 Ronquist F, Teslenko M, van der Mark P, Ayres DL, Darling A, Höhna S, Larget B, Liu L,

697 Suchard MA, Huelsenbck JP. 2012. MrBayes 3.2: efficient Bayesian phylogenetic inference and 698 model choice across a large model space. Systematic Biology 61(3):539-542.

699 doi:10.1093/sysbio/sys029.

700 Salzberg SL, Deicher AL, Kasif S, White O. 1998. Microbial gene identification using 701 interpolated Markov models. Nucleic Acids Research 26(2):544-548. doi:

702 https://doi.org/10.1093/nar/26.2.544.

703 Sambrook JR, Russell DW. 2001. Molecular cloning: a laboratory manual. Cold Spring Harbor 704 Laboratory Press, New York.

705 Sanssouci E, Lerat S, Grondin G, Shareck F, Beaulieu C. 2011. Tdd8: a TerD domain-encoding 706 gene involved in Streptomyces coelicolor differentiation. Antonie van Leeuwenhoek

707 100(3):385-398. doi: 10.1007/s10482-011-9593-y. 
708 Seemann T. 2014. Prokka: rapid prokaryotic genome annotation. Bioinformatics

709 30(14):2068-2069. doi: 10.1093/bioinformatics/btu153.

710 Simão FA, Waterhouse RM, Ioannidis P, Kriventseva EV, Zdobnov EM. 2015. BUSCO:

711 assessing genome assembly and annotation completeness with single-copy orthologs.

712 Bioinformatics 31(19):3210-3212. doi: 10.1093/bioinformatics/btv351.

713 Tamura K, Stecher G, Peterson D, Filipski A, Kumar S. 2013. MEGA6: molecular evolutionary

714 genetics analysis version 6.0. Molecular Biology and Evolution 30(12):2725-2729. doi:

$71510.1093 / \mathrm{molbev} / \mathrm{mst} 197$.

716 Tanenbaum DM, Goll J, Murphy S, Kumar P, Zafar N, Thiagarajan M, Madupu R, Davidsen T, 717 Kagan L, Kravitz S, Rusch DB, Yooseph S. 2010. The JCVI standard operating procedure for 718 annotating prokaryotic metagenomic shotgun sequencing data. Standards in Genomic Sciences 719 2(2):229-237. doi: 10.4056/sigs.651139

720 Taylor DE. 1999. Bacterial tellurite resistance. Trends in Microbiology 7(3):111-115.

721 Taylor DE, Rooker M, Keelan M, Ng LK, Martin I, Perna NT, Burland NT, Blattner FR. 2002.

722 Genomic variability of $\mathrm{O}$ islands encoding tellurite resistance in enterohemorrhagic Escherichia

723 coli O157: H7 isolates. Journal of Bacteriology 184(17):4690-4698.

724 Taylor DE, Walter EG, Sherburne R, Bazett-Jones DP. 1988. Structure and location of tellurium 725 deposited in Escherichia coli harbouring tellurite resistance plasmids. Journal Ultrastructure 726 and Molecular Structure Research 99(1):18-26.

727 Toptchieva A, Sisson G, Bryden LJ, Taylor DE, Hoffman PS. 2003. An inducible tellurite-

728 resistance operon in Proteus mirabilis. Microbiology 149(Pt 5):1285-1295. doi:

729 10.1099/mic.0.25981-0.

730 Tritt A, Eisen JA, Facciotti MT, Darling AE. 2012. An integrated pipeline for de novo assembly 731 of microbial genomes. PLoS ONE 7(9):e42304. doi:10.1371/journal.pone.0042304.

732 Tuner S, Pryer KM, Miao VPW, Palmer JD. 1999. Investigating deep phylogenetic relationships

733 among cyanobacteria and plastids by small submit rRNA sequence analysis. Journal of

734 Eukaryotic Microbiology 46(4):327-338. doi: 10.1111/j.1550-7408.1999.tb04612.x.

735 Turkovicova L, Smidak R, Jung G, Turna J, Lubec G, Aradska J. 2016. Proteomic analysis of the 736 TerC interactome: novel links to tellurite resistance and pathogenicity. Journal of Proteomics

737 136:167-73. doi: 10.1016/j.jprot.2016.01.003.

738 Turner RJ, Borghese R, Zannoni D. 2012. Microbial processing of tellurium as a tool in

739 biotechnology. Biotechnology Advances 30(5):954-963. doi: 10.1016/j.biotechadv.2011.08.018.

740 Varghese NJ, Mukherjee S, Ivanova N, Konstantinidis KT, Mavrommatis K, Kyrpides NC, Pati 741 A. 2015. Microbial species delineation using whole genome sequences. Nucleic Acids Research 742 43(14):6761-6771.doi: 10.1093/nar/gkv657. 
743 Whelan KF, Colleran E. 1992. Restriction endonuclease mapping of the HI2 incompatibility 744 group plasmid R478. Journal of Bacteriology 174(4):1197-1204.

745 Whelan KF, Colleran E, Taylor DE. 1995. Phage inhibition, colicin resistance, and tellurite 746 resistance are encoded by a single cluster of genes on the IncHI2 plasmid R478. Journal of 747 Bacteriology 177(7):5016-5027.

748 Whelan KF, Sherburne RK, Taylor DE. 1997. Characterization of a region of the IncHI2 plasmid R478 which protects Escherichia coli from toxic effects specified by components of tellurite, phage and colicin resistance cluster. Journal of Bacteriology 179(1):63-71.

751

752

753

754

755

756

757

758

759

760

761

762

763

764

765

766

767

768

769

770

771

772

773

774

775

776

777

Wickham H. 2007. Reshaping Data with reshape Package. Journal of Statistical Software. 21(12): $1-20$.

Wu M, Eisen JA. 2008. A simple, fast, and accurate method of phylogenetic inference. Genome Biology 9(10):R151. doi: 10.1186/gb-2008-9-10-r151.

(1)

\section{Figure legends}

Figure 1. Phylogenetic, morphological and genomic characteristics of $\boldsymbol{P}$. glacincola BNF20. (A) Scanning electron micrograph showing the morphology and dimensions of $P$. glacincola BNF20. Samples were stained with uranyl acetate $(0.5 \% \mathrm{w} / \mathrm{v})$ and examined using a low-voltage electron microscope (Delong Instruments, LVEM5), with a nominal operating voltage of $5 \mathrm{kV}$. Bar represents 10 $\mu \mathrm{m}$. (B) Phylogenetic tree of P. glacincola BNF20 based on the partial 16S rRNA gene sequence (Accession number MF806171). Psychrobacter ingroup was rooted using Moraxella osloensis DSM $6978^{\mathrm{T}}$ as outgroup. (C) Circular map of the 18-scaffold draft genome with coding sequences colored by COG categories. Inner circles represent GC Skew and GC content.

Figure 2. Whole genome nucleotide identity and multi-locus phylogenetic analysis. (A) Average nucleotide identity (ANI) in the 35-genome Psychrobacter dataset. P. glacincola BNF20 forms a cluster with other three Psychrobacter genomes with an alignment fraction over $80 \%$. (B) Bayesian multi-locus phylogenetic analysis of the genomic sequences from the indicated Psychrobacter members. Taxa are colored by geographic location. Node values correspond to posterior probabilities, and the phylogeny was mid-point rooted.

Figure 3. Genomic context of the ter genes harbored by $\boldsymbol{P}$. glacincola BNF20. (A) Context of the gene cluster located at nucleotide (nt) positions 189,803-204,267 of the Contig LIQB1000002.1. (B) Contig LIQB01000003.2, located at nt positions 286,133-301,767 exhibits an extra copy of the terZ gene. 
778 Figure 4. Number of ter genes in Bacterial families. Distribution of ter genes present in the indicated 779 phyla. Only taxonomic classifications (Phylum and Family) with at least 3 bacterial genomes encoding at 780 least one ter gene are shown. 


\section{Table 1 (on next page)}

Classification and general features of $P$. glacincola BNF20. 
1

2 Table 1. Classification and general features of P. glacincola BNF20.

3

\begin{tabular}{|c|c|c|}
\hline MIGS ID & Property & Term \\
\hline & Classification & Domain: Bacteria \\
\hline & & Phylum: Proteobacteria \\
\hline & & Class: Gammaproteobacteria \\
\hline & & Order: Pseudomonadales \\
\hline & & Family: Moraxellaceae \\
\hline & & Genus: Pychrobacter \\
\hline & & Species: Psychrobacter glacincola \\
\hline & & (Type) strain: BNF20 \\
\hline & Gram stain & Negative \\
\hline & Cell shape & Rod \\
\hline & Motility & Non-Motile \\
\hline & Sporulation & Non-sporulating \\
\hline & Temperature range & Psychrotolerant \\
\hline & $\begin{array}{l}\text { Optimum } \\
\text { temperature }\end{array}$ & $25^{\circ} \mathrm{C}$ \\
\hline & pH range; Optimum & Not tested; 7.4 \\
\hline & Carbon source & Citrate, acetate, pyruvate \\
\hline MIGS-6 & Habitat & Antarctic sediment \\
\hline MIGS-6.3 & Salinity & $0-10 \% \mathrm{NaCl}(\mathrm{w} / \mathrm{v})$ \\
\hline MIGS-22 & Oxygen requirement & Aerobic \\
\hline MIGS-15 & Biotic relationship & Free-living \\
\hline MIGS-14 & Pathogenicity & Potentially pathogenic \\
\hline MIGS-4 & Geographic location & King George Island, Antarctica \\
\hline MIGS-5 & Sample collection & January, 2012 \\
\hline MIGS-4.1 & Latitude & $62^{\circ} 11^{\prime} \mathrm{S}$ \\
\hline MIGS-4.2 & Longitude & $58^{\circ} 56^{\prime} \mathrm{W}$ \\
\hline MIGS-4.4 & Altitude & Not registered \\
\hline
\end{tabular}

4

5

6

7

8

9

10

11

12

13

14

15 


\section{Table 2 (on next page)}

Minimal inhibitory concentrations (mM) of different metal(loid)s for P. glacincola BNF20, and E. coli BW25113. 
Table 2. Minimal inhibitory concentrations (mM) of different metal(loid)s for P. glacincola BNF20, and E. coli BW25113 (reference).

\begin{tabular}{ccc}
\hline Metal & $\mathrm{BNF} 20$ & $\mathrm{BW} 25113$ \\
\hline $\mathrm{TeO}_{3}{ }^{2-}$ & 2.3 & 0.004 \\
\hline $\mathrm{Cu}^{2+}$ & 3.12 & 6 \\
\hline $\mathrm{Cd}^{2+}$ & 0.062 & 1 \\
\hline $\mathrm{Hg}^{2+}$ & 0.0039 & 0.01 \\
\hline $\mathrm{Zn}^{2+}$ & 0.5 & 2 \\
\hline $\mathrm{CrO}_{4}{ }^{2-}$ & 6 & 1.5 \\
\hline $\mathrm{AuCl}_{4}{ }^{1-}$ & 0.015 & 0.16 \\
\hline $\mathrm{Ni}^{2+}$ & 1.25 & 5 \\
\hline $\mathrm{AsO}_{4}{ }^{2-}$ & 40 & 80 \\
\hline $\mathrm{AsO}_{2}{ }^{1-}$ & 5 & 10 \\
\hline $\mathrm{Ag}^{1+}$ & 0.015 & 0.063 \\
\hline
\end{tabular}




\section{Figure $\mathbf{1}$ (on next page)}

Phylogenetic, morphological and genomic characteristics of $P$. glacincola BNF20.

(A) Scanning electron micrograph showing the morphology and dimensions of $P$. glacincola BNF20. Samples were stained with uranyl acetate $(0.5 \% \mathrm{w} / \mathrm{v})$ and examined using a lowvoltage electron microscope (Delong Instruments, LVEM5), with a nominal operating voltage of $5 \mathrm{kV}$. Bar represents $10 \mu \mathrm{m}$. (B) Phylogenetic tree of $P$. glacincola BNF20 based on the partial 16S rRNA gene sequence (Accession number MF806171). Psychrobacter ingroup was rooted using Moraxella osloensis DSM 6978T as outgroup. (C) Circular map of the 18-scaffold draft genome with coding sequences colored by COG categories. Inner circles represent GC Skew and GC content. 
A)

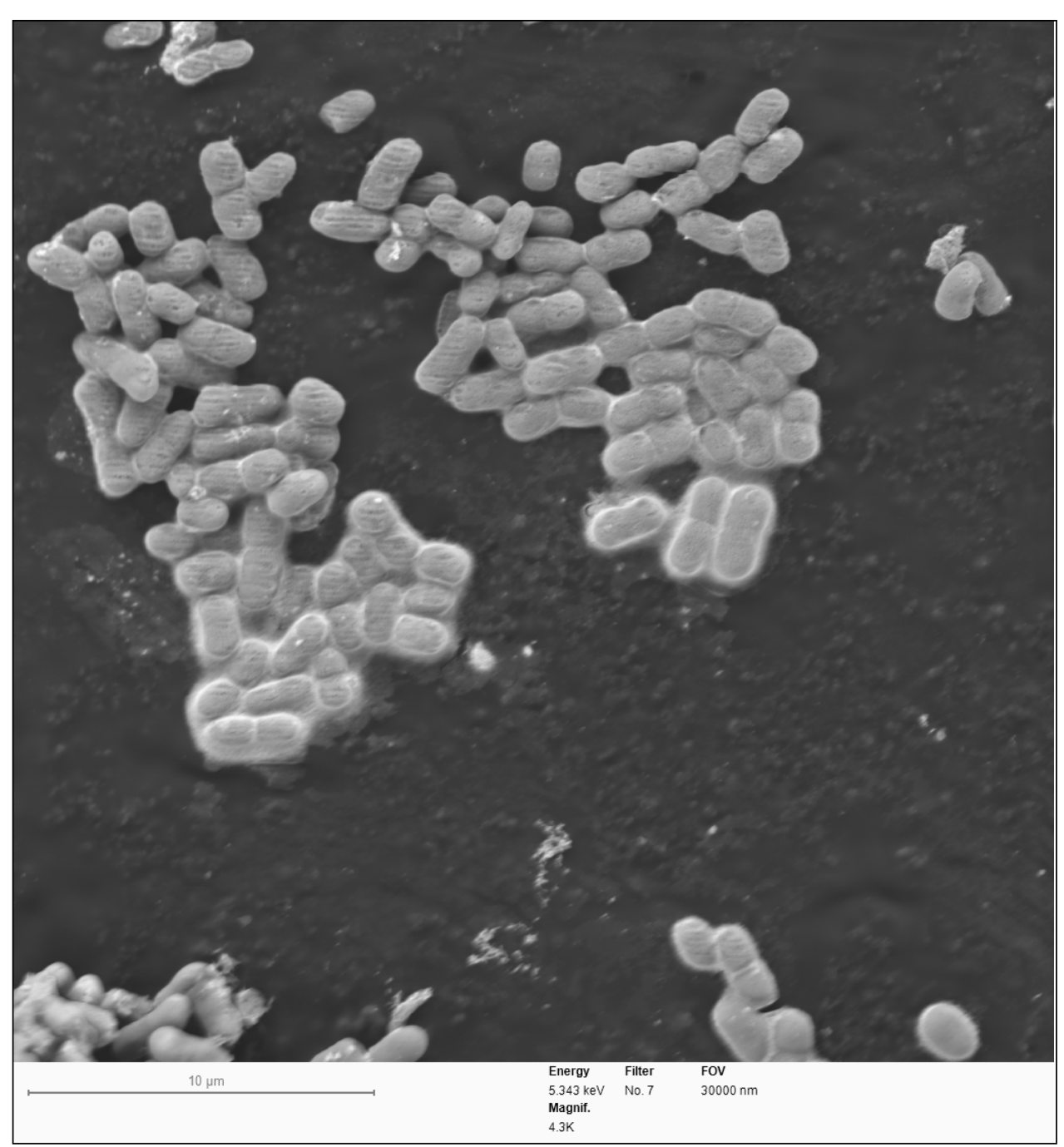

B)

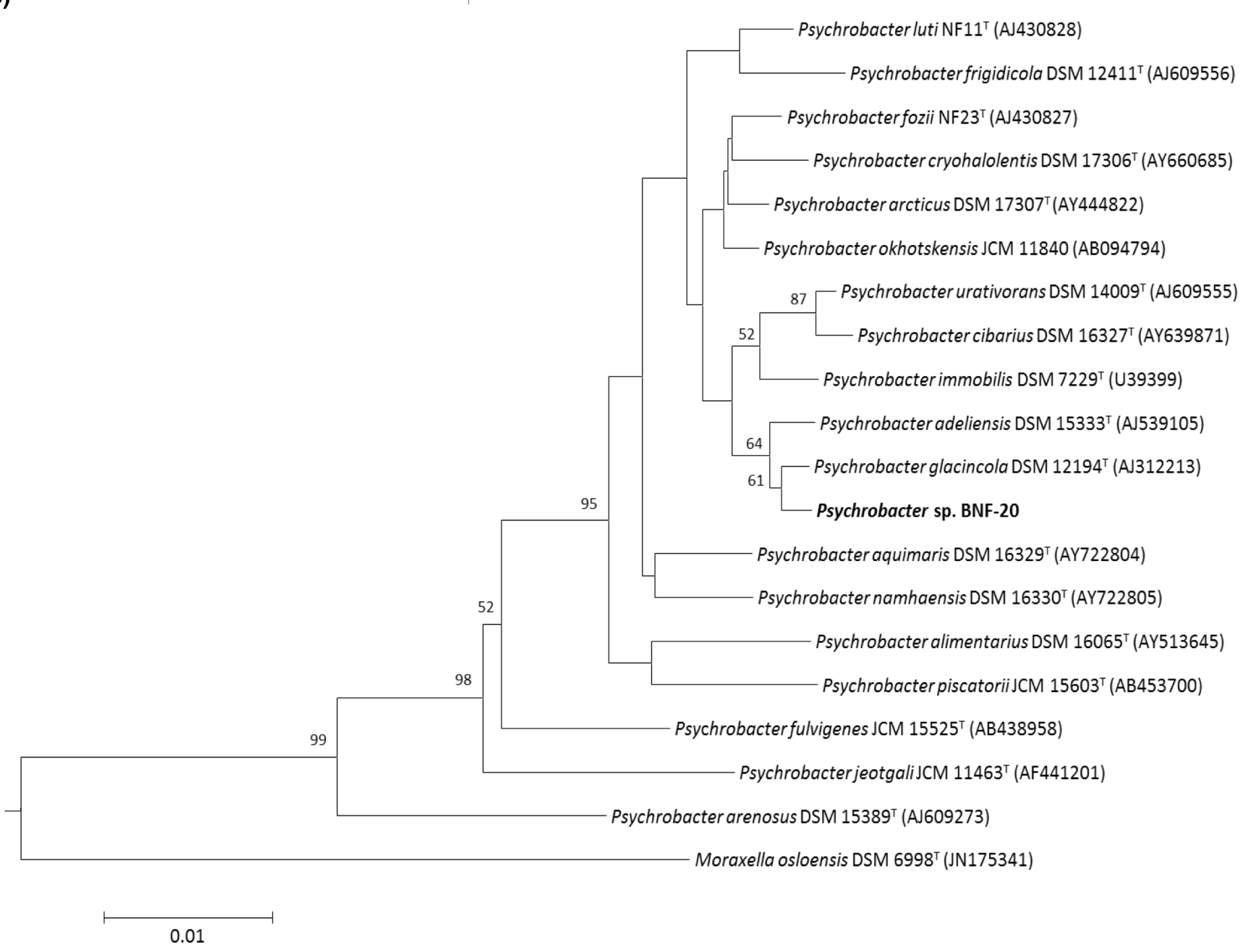

C)

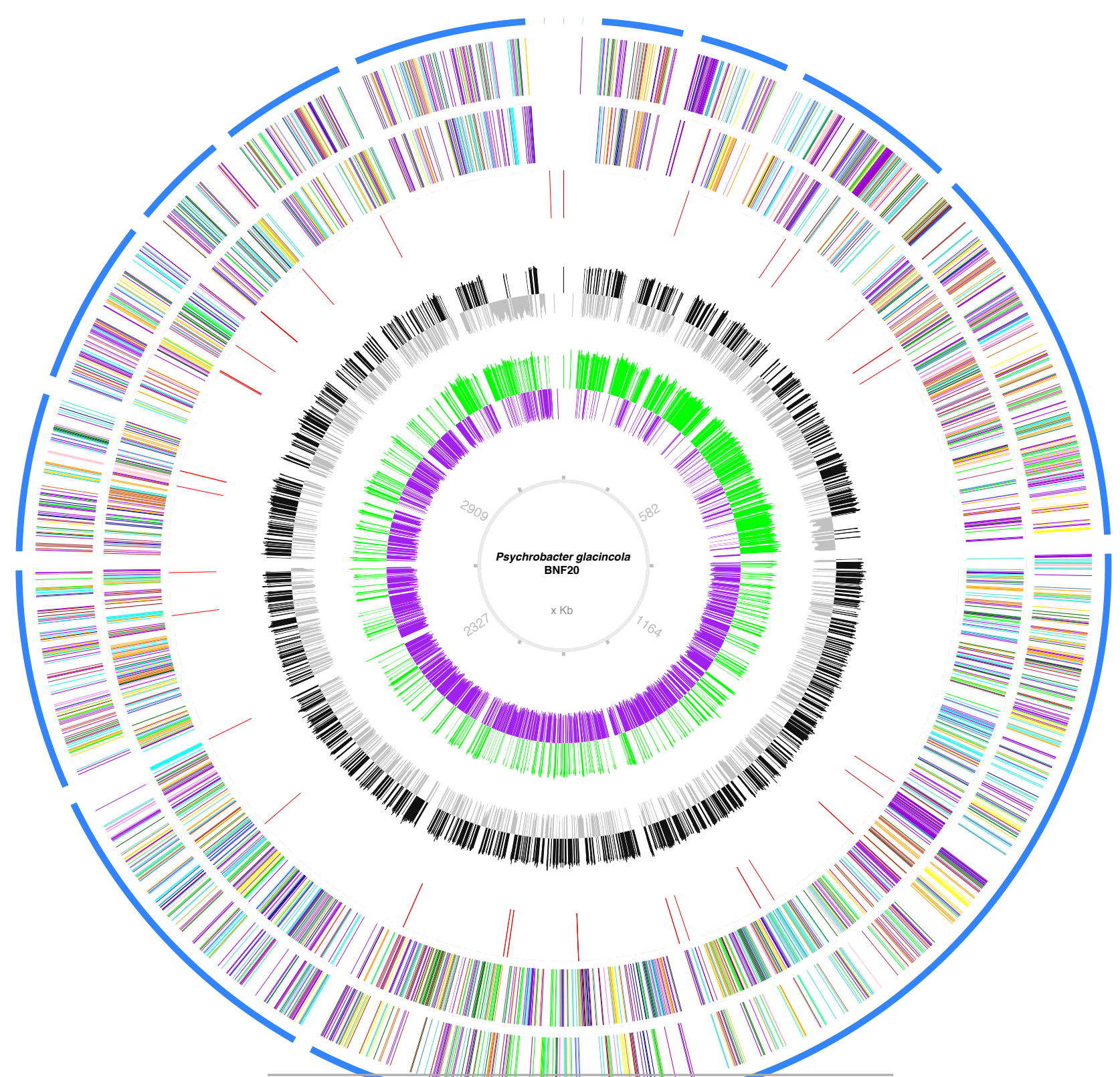

COG Categories

- RNA processing and modification

- Energy production and conversion

Cell cycle control, cell division, chromosome partitioning

- Amino acid transport and metabolism

Nucleotide transport and metabolism

- Carbohydrate transport and metabolism

- Coenzyme transport and metabolism

- Lipid transport and metabolism

- Translation, ribosomal structure and biogenesis

- Transcription

- Replication, recombination and repair

- Cell wall/membrane/envelope biogenesis

- Cell motility

- Post-translational modification, protein turnover, chaperones

- Inorganic ion transport and metabolism

Secondary metabolites biosynthesis, transport and catabolism

- General function prediction only

- Function unknown

- Signal transduction mechanisms

Intral transducton mechanisms

- Defense mechanisms

- Cytoskeleton

- tRNA

- rRNA

- GC Skew +

GC Skew

Peer) reviewing PDF | (2017:10:21147:2:0:NEW 5 Jan 2018) 
Figure 2 (on next page)

Whole genome nucleotide identity and multi-locus phylogenetic analysis.

(A) Average nucleotide identity (ANI) in the 35-genome Psychrobacter dataset. P. glacincola BNF20 forms a cluster with other three Psychrobacter genomes with an alignment fraction over $80 \%$. (B) Bayesian multi-locus phylogenetic analysis of the genomic sequences from the indicated Psychrobacter members. Taxa are colored by geographic location. Node values correspond to posterior probabilities, and the phylogeny was mid-point rooted. 


\section{Figure 3 (on next page)}

Genomic context of the ter genes harbored by P. glacincola BNF20.

(A) Context of the gene cluster located at nucleotide (nt) positions 189,803-204,267 of the Contig LIQB1000002.1. (B) Contig LIQB01000003.2, located at nt positions 286,133-301,767 exhibits an extra copy of the ter $Z$ gene. 


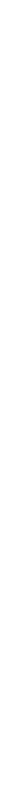


Figure 4 (on next page)

Number of ter genes in Bacterial families.

Distribution of ter genes present in the indicated Phyla. Only taxonomic classifications (Phylum and Family) with at least 3 bacterial genomes encoding at least one ter gene are shown. 
Nitrosomonadaceae (5) Ectothiorhodospiraceae (4) -

Methylobacteriaceae (19)

Rhodospirillaceae (10) -

Rickettsiaceae (16) -

Legionellaceae (15) -

Hyphomicrobiaceae (11)

Yersiniaceae (13)

Phyllobacteriaceae (13)

Methylococcaceae (13)

Halomonadaceae (13)

Burkholderiaceae (13)

Morganellaceae (12)

Caulobacteraceae (11)

Aurantimonadaceae (11)

Acetobacteraceae (11)

Bradyrhizobiaceae (10)

Aeromonadaceae (10)

Sphingomonadaceae (45)

Moraxellaceae (45) -

Vibrionaceae (42) -

Comamonadaceae (39) -

Xanthomonadaceae (37) -

Enterobacteriaceae (37) -

Pseudomonadaceae (31) -

Rhizobiaceae (29) -

Oxalobacteraceae (25) -

Rhodobacteraceae (24) -

Neisseriaceae (24) -

Erwiniaceae (24) -

Chromobacteriaceae (21) -

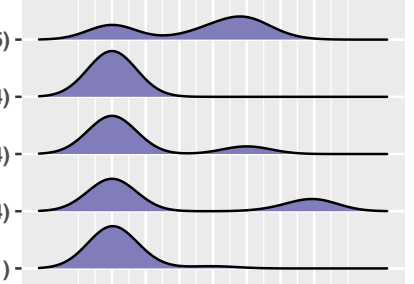

$\begin{array}{lllllll}1 & 2 & 3 & 4 & 5 & 1 & 1\end{array}$

\section{Peareti}

Pasteurellaceae (4) Methylocystaceae (4) -

Thiobacillaceae (3)

Rhodocyclaceae (3) -

Psychromonadaceae (3) -

Cellvibrionaceae (3)

Cardiobacteriaceae (3)

Budviciaceae (3)

Brucellaceae (3)

Zoogloeaceae (10)

Pectobacteriaceae (9)

Chromatiaceae (9) -

Alcaligenaceae (9)

Erythrobacteraceae (8)

Shewanellaceae (7)

Alteromonadaceae (6) -

Thiotrichaceae (5)

Oceanospirillaceae (5)

Streptomycetaceae (149) -

Microbacteriaceae (86)

Corynebacteriaceae (72) -

Mycobacteriaceae (55) -

Micrococcaceae (55) -

Pseudonocardiaceae (48) -

Nocardiaceae (45) -

Bifidobacteriaceae (36) -

Actinomycetaceae (36) -

Nocardioidaceae (35) -

Intrasporangiaceae (26) -

$\begin{array}{lllllll}2 & 3 & 4 & 5 & 6 & 7\end{array}$
Promicromonosporaceae (4):- Manys Civit to be reviewed

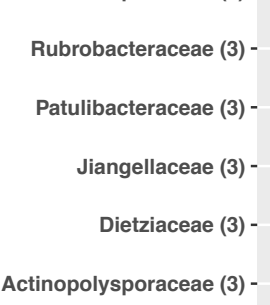

Actinopolysporaceae (3) -

Frankiaceae (8) -

Thermomonosporaceae (7)

Demequinaceae (7) -

Brevibacteriaceae (7)

Dermabacteraceae (6)

Glycomycetaceae (5) -

Dermatophilaceae (4) -

Micromonosporaceae (20) -

Gordoniaceae (20) -

Nocardiopsaceae (18) -

Propionibacteriaceae (16) -

Cellulomonadaceae (16) -

Streptosporangiaceae (10) -

Eubacteriaceae (4) -

Carnobacteriaceae (4) -

Ruminococcaceae (10) -

Peptococcaceae (10) -

Bacillaceae (84) -

Lactobacillaceae (82) -

Paenibacillaceae (39) -

Clostridiaceae (39) -

Lachnospiraceae (22) -

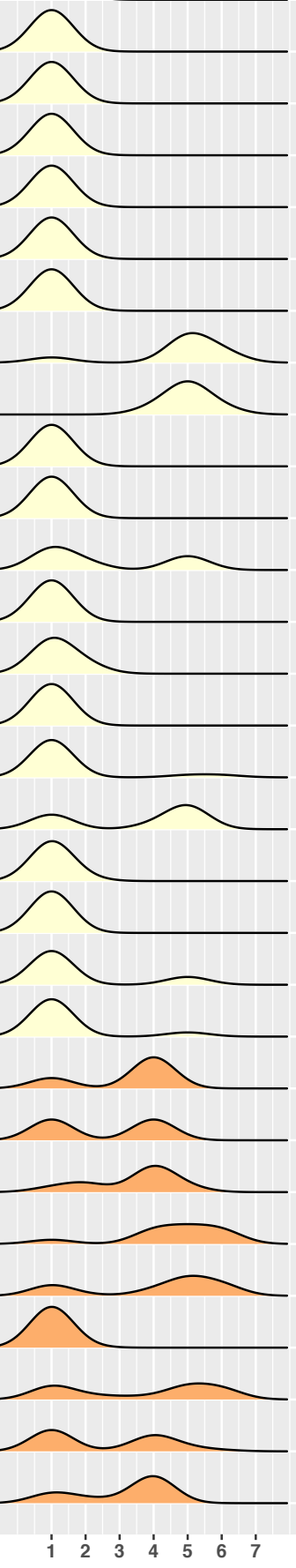

Dermacoccaceae (6)
Staphylococcaceae (4) -

Sporomusaceae (4) -

Listeriaceae (4)

Thermosinomyetacene (3)-

Selenomonadaceae (3) -

Enterococcaceae (3) -

Planococcaceae (9) -

Peptostreptococcaceae (8) -

Leuconostocaceae (8)

Flammeovirgaceae (7) -

Cyclobacteriaceae (7) -

Cytophagaceae (20)

Hymenobacteraceae (15) -

Prevotellaceae (14)-

Chitinophagaceae (11) -

Flavobacteriaceae (114)

Sphingobacteriaceae (22) -

Oscillatoriaceae (3) -

Microcoleaceae (5)

Dehalococcoidaceae (3) -

Chloroflexineae (4) -

Thermaceae (5)

Deinococcaceae (15)

Planctomycetaceae (3)

Chlorobiaceae (3) -

Aquificaceae (3)

Methylacidiphilaceae (3) -

Methanomassililicoccaceae (4)

Acidobacteriaceae (10) -

Spirochaetaceae (3) -

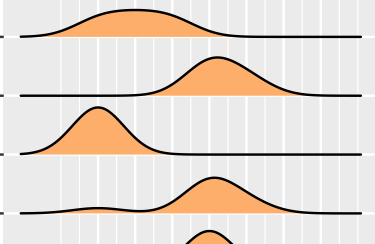

$\square$ Proteobacteria

Actinobacteria

Firmicutes

Bacteroidetes

Cyanobacteria

Deinococcus-Thermus

Chloroflexi

Spirochaetes

Acidobacteria

Euryarchaeota

Verrucomicrobia

Aquilicae

Chlorobi

Planctomycetes 